\title{
Development of a miniature robot for swarm robotic application
}

\begin{abstract}
Biological swarm is a fascinating behavior of nature that has been successfully applied to solve human problem especially for robotics application. The high economical cost and large area required to execute swarm robotics scenarios does not permit experimentation with real robot. Model and simulation of the mass number of these robots are extremely complex and often inaccurate. This paper describes the design decision and presents the development of an autonomous miniature mobile-robot (AMiR) for swarm robotics research and education. The large number of robot in these systems allows designing an individual AMiR unit with simple perception and mobile abilities. Hence a large number of robots can be easily and economically feasible to be replicated. AMiR has been designed as a complete platform with supporting software development tools for robotics education and researches in the Department of Computer and Communication Systems Engineering, UPM. The experimental results demonstrate the feasibility of using this robot to implement swarm robotic applications.
\end{abstract}

Keyword: Autonomous; AMiR; Swarm intelligent; Low-cost; Platform; Education 